\title{
Issues of physical interaction of unmanned aircraft manipulators with ground objects
}

\author{
Vinh Nguyen ${ }^{1, *}$, Oksana Solenaya ${ }^{1}$ and Petr Smirnov ${ }^{2}$ \\ ${ }^{1}$ SUAI, Department of Electromechanics and Robotics, 190000, Saint-Petersburg, Bolshaya Morskaya Str., 67, Russia \\ ${ }^{2}$ SPIIRAS, Laboratory of Autonomous Robotic Systems, 199178, Saint-Petersburg, 14-th line of V.I., 39, Russia
}

\begin{abstract}
Adding an onboard manipulation system to an unmanned aerial vehicle (UAV) significantly complicates framework, functioning algorithms, and leads to an increase in overall dimensions. The physical interaction of the manipulator with objects influences to unstabilization of UAV, which in turn leads to difficulties in positioning the UAV and reduces the accuracy of gripper motion. In addition, the physical interaction of the manipulator with objects requires increased power resources of UAVs. The article analyzes modern research of UAVs with a manipulator, including flight control problems, avoidance of contact with the earth, surrounding space, as well as manipulations with the captured object. On the basis of the analysis, a list of new problems arising in the physical interaction of UAVs with objects through an embedded manipulator is formulated.
\end{abstract}

\section{Introduction}

At present, unmanned aerial vehicles (UAVs) are increasingly being used to solve problems of monitoring various types of objects and territories. Also, a promising area of research is the transportation of goods through UAVs, the main advantage of which in the urbanized area is high speed, while strict security requirements are imposed. UAVs should avoid obstacles, do not slow down or interfere with the movement of land vehicles. Unlike fixed-wing aircraft, rotorcraft (FWA), such as helicopters and multicopters, can travel through narrow aisles. The FWA can also hang and observe what is useful when interacting with stationary objects. Some FWA can land in the course of the task to prolong battery life and reduce power consumption, for example, when viewed from a stationary site. In work [1] approaches to the solution of the problem of increasing the operating time of unmanned aerial vehicles in autonomous missions based on charge or replacement of on-board batteries on the accompanying robotic platform are analyzed.

Recent advances in global and local navigation, localization, detection and prevention of obstacles have significantly increased the autonomy of UAVs. Stabilization of flight parameters and constant monitoring of surrounding objects are important aspects in autonomous operations performed by UAVs. Most of the research to date has focused on developing robust autopilot systems, automated workplaces for pilot-toaircraft interaction using 3D modeling and virtual reality systems, and analyzing safety issues, including preventing the drones from collapsing.

The ability of aerial unmanned vehicles to manipulate or carry surrounding objects greatly expands the types of missions and applications, enhancing the operator's capabilities in performing dirty, dangerous or monotonous operations such as: manipulation of objects, building structures, repair of building infrastructures, assistance in natural disasters, loading cargo, inspection and maintenance of industrial plants, taking samples of materials from hard-to-reach areas, inspection of transport, agricultural land and other uses. Such typical tasks can be: collection of soil samples, maintenance on the bridge or on the facade of the building, cleaning of debris in the danger zone. In such tasks, the UAV must be able to manipulate in its environment, including removing obstacles that block movement to the target point. However, at present only ground-based robotic vehicles are equipped with manipulators $[2,3]$.

The control systems of the multi-rotor platforms equipped with a manipulator with a grip are also called MM-UAV (Mobile Manipulating Unmanned Aerial Vehicles). The development of unmanned aircraft manipulators (UAM) is accompanied by a much greater number of difficulties than the creation of traditional ground-based robots that perform the tasks of movement together with manipulation. The most difficult issue is the lack of a stable platform in the air. Ground vehicles can stop to perform operations and in a stable state to perform precise manipulations, while UAMs in most cases do not have this capability. In fact, even with the use of robust flight stabilization systems, the UAM will be in a certain area of space, without having precise fixed coordinates, especially when operating in an open space. The lack of stability of the air platform can be partially compensated by manipulator control. The range of manipulator movements and the field of vision sensors also depend on the side with which the platform

Corresponding author: nguyenvanvinhhvkt@gmail.com 
flies to the object. The workspace of the manipulator is limited by the body of the air platform itself, so to increase it in most cases the manipulator is mounted directly to the lower part of the platform [4].

Thus, the focus of previous research was the solution of the problem of flight control, avoidance of contact with the earth and the surrounding space. The purpose of this study is the physical interaction of UAVs with objects through an integrated manipulator. The following is an overview of the main problems encountered by unmanned aircraft manipulator developers and the analysis of published results. It should be noted that experiments on full-scale models are still rarely carried out, mainly computer or combined modeling, where the model of the manipulator is suspended in a special frame that simulates the free movement of the UAV. In the last section, a list of tasks that arise in the development of unmanned aircraft manipulators, not studied previously in the design of UAVs and ground-based manipulators, is formulated.

\section{Problems of development of unmanned aerial manipulators and analysis of existing solutions}

Interacting with objects using a manipulator mounted on unmanned aerial vehicles is an important task, since UAVs can access many places that are in some cases inaccessible to other ground transport robotic platforms [5]. However, adding an on-board manipulation system to a flying robot causes serious problems, as operations of physical interaction with ground objects affect the overall stability of the entire air platform. During the flight of an UAV with a moving mobile manipulation system, the mass distribution changes and additional dynamic reaction forces arise [6]. The design of the mechanism for capturing and manipulating objects affects the permissible mass of the payload, the inertial and dynamic characteristics of the entire UAV. It is important that the mass of this mechanism is low and its center of mass is closer to the base of the $\mathrm{UAV}$, in this case the emerging reaction forces and torque that will arise during the UAV movement will not significantly affect its stability. The effect of propagating contact forces from the final grab to the UAV can be minimized through the connection of the air manipulator with the UAV base [7]. Such aspects are usually not taken into account in standard algorithms of platform stabilization, so a new algorithm is needed that solves such problems. It should also be noted that manipulators perform various types of movements, translational, rotating, with a payload and without, which causes various effects on UAVs that violate its stability [6].

It is also necessary to analyze a number of other serious problems before it becomes possible to manipulate objects through UAVs, among which [5] are distinguished: 1) the UAV platform constantly has micropropulsion and, accordingly, it is extremely difficult to accurately position the links of the UAV manipulator relative to the object of interest for manipulation; 2) manipulator movements affect the stability of the UAV platform, which further complicates positioning.
In work [4], the authors have constructed a minigantry test rig and built a simulation environment in OpenRAVE for a dual arm UAV manipulator system. The authors envision Mobile Manipulator UAV system performing similar tasks as existing ground robots, but with a higher degree of autonomy, speed, and mobility. The design consists of two manipulators that are mounted to the bottom of the mini-gantry crane. The authors focus on dual arm manipulation and grasping tasks. Attached to the arms are 1-DOF Melissa hands consisting of four fingers and a thumb. As a result, the forces of reaction that the UAV experiences during manipulation when pointing to an object were investigated. A model for compensating this force and ensuring the quasi-stability of UAVs at contact with the object is created.

In work [5], a multi-link manipulator was installed on a platform with 3 degrees of freedom to simulate the linear motions of the Ascending Technologies Pelican quadrotor. Angles by rolls, pitch and yaws were not applied in emulated movements due to the mechanical complexity of the implementation. The manipulator is assembled from servomotors and passive elements. Reconfigurable capture with two degrees of freedom and one drive allows you to take a variety of objects with minimal movements. A preliminary demonstration of the manipulator's capabilities was performed on an example of simulating the task of removing and installing insulators from high-voltage power lines.

In work [7], has presented the design and experimental validation of a compliant and low weight (40 grams) anthropomorphic finger module specifically designed for aerial manipulation and grasping. The three joints of the finger are under actuated and driven by a nylon tendon that is rolled up on a small section reel attached to a micro motor shaft The anthropomorphic finger consists in three $8 \mathrm{~mm} \mathrm{U}$ shape aluminum profile sections of $45 \mathrm{~mm}$ (proximal phalange), $20 \mathrm{~mm}$ (middle phalange) and $15 \mathrm{~mm}$ (distal phalange) length. The finger module is capable of grasping objects of different shapes and forms in a stable way by itself, without requiring any other fingers. If the finger is not grasping any object, it can be used for detecting and reacting against collisions of the manipulator with the environment in a safe way as joint compliance will cause the passive contraction of the finger. This may be especially useful in the navigation of the aerial platform in narrow areas. For this purpose, the finger would remain extended and the metacarpophalangeal joint position controller disabled.

In work [8], The MM-UAV prototype is constructed using a low-cost quadrotor-type aircraft, a GAUI 330, and three manipulator arms. The GAUI quadrotor uses four equally positioned brushless motors equipped with 8-inch propellers to provide lift and maneuverability. The total vehicle diameter is $330 \mathrm{~mm}$ and the total weight is approximately $400 \mathrm{~g}$ with a maximum flying weight of $1100 \mathrm{~g}$. The overall weight of the aircraft is kept bellow $950 \mathrm{~g}$, thus making the aircraft capable of lifting small objects bellow $200 \mathrm{~g}$. The final construction and important construction points (i.e. Center of Assembly-COA, Center of Mass of manipulator arm- COM, and Center of Gravity of aircraft-COG) are appropriately marked. 
Each arm of the manipulator has 2 degrees of freedom. For additional manipulation capabilities at the end, the manipulator is equipped with a hook-to-hook, push / pull objects from the floor. The simulation results showed that the manipulator creates the least amount of UAV interference in the case when all the links move simultaneously. Nevertheless, experimental results show that the multicopter has oscillations.

In work [9], a multicopter equipped with a manipulator weighing $200 \mathrm{~g}$, capturing a payload of up to $200 \mathrm{~g}$, is described. The multicopter itself can carry a payload in excess of $500 \mathrm{~g}$ (greater than the total weight of the manipulator with payload). In the developed controller, three control methods were used to move the UAV: the linear feedback method (FL-PID), the direct fuzzy control method (DFLC) and the fuzzy model control method (FMRLC). The methods were tested in the capture of the payload and release from it. The FL-PID method did not ensure the stability of the UAV when increasing the payload and there were delays in performing real-time operations. The DFLC and FMRLC method allowed to maintain the stability of the UAV when adding / removing from the payload, while the FMRLC method showed a higher positioning accuracy.

In work [10] the problem of the accuracy of navigation and motion control of several connected UAVs with manipulators was investigated, a general structure of modeling of aviation vehicles with several fuselages was developed. To demonstrate the proposed models as a separate element used a hexagonal UAV, equipped with a simple manipulator.

In work [11] the helicopter SST-Eagle2-GS Long Tail, released by $\mathrm{HIROBO}$, equipped with a manipulator, is described. During the tests, the effect of the added weight (manipulator) on the parameters of the helicopter control system was studied. Taking into account that the added weight of the manipulator changed the total weight of the helicopter by $25 \%$, the MP2128Heli autopilot equipped with PID controllers did not provide stability and when testing shortly after takeoff the helicopter heared into lateral fluctuations, therefore the algorithm for calculating the PID controller values was modified and with new parameters was provided stable helicopter flight with additional weight. The testing was carried out with the helicopter hanging autonomously at a height of $3 \mathrm{~m}$.

In work [14] a successful application of a quadrotor with a two-DOF robot arm attached for picking up and delivering an object. Kinematic and dynamic models for the combined system are analyzed and an adaptive sliding mode controller is designed to control them all together. This is demonstrated in an experiment with a scenario consisting of picking up an object, moving, and releasing it inside of a shelf. The experimental results demonstrates satisfactory performance to perform the designed tasks. The proposed aerial manipulation using the adaptive sliding mode controller is validated by an experiment.

The first mission for the combined system is picking up an object. The object used in the experiment is an wood block of dimension (H) 7.5x (D) $5 x$ (W) $1.5 \mathrm{~cm}$. Given that the gap of the end effector is only $3.2 \mathrm{~cm}$, it is clear that grabbing the wood block requires accurate control of the quadrotor and robot arm concurrently.
Even though no information such as mass and moment of inertia about the wood block is given to the adaptive sliding mode controller, overall manipulation is successful because of robustness of the sliding mode controller. During the pick-up phase, root-mean-square error of the quadrotor position is only $2.08 \mathrm{~cm}$. The second mission is releasing the wood block inside a shelf. The size of the gap of shelf is about $(\mathrm{H}) 16 \times(\mathrm{W})$ $22 \mathrm{~cm}$. To lay the wood block down inside the shelf, most part of the second link with gripper needs to be put into the shelf. Again, given the size of the second link with respect to the size of the shelf, it is a difficult task that demands exact control of the combined system.

From the entire trajectory histories, it is shown that the quadrotor and robotic arm are controlled as desired during the full flight including manipulations of an object. These results also means that the provided approach can be utilized in expanded manipulation and transportation applications.

In work [15] presented a method for planning a timeoptimal trajectory for a quadrotor equipped with an onboard gripper with the goal of grasping a moving target while traveling between generic initial and final states. We introduced and used two complementary set of trajectories able to continuously keep the quadrotor gripper in contact with the moving target. The method takes into account upper/lower bounds on the propeller speeds and the need of a finite time for the gripper in order to successfully perform the grasping task. Extensive physically-based simulations demonstrate the effectiveness of the proposed strategy.

With respect to the state of the art, the contributions of this paper can be summarized as follows: i) we explicitly consider the duration of the trajectory in order to minimize it; ii) we allow the quadrotor to attain generic, and in particular non-hovering, states for starting and ending the grasping phase; iii) we take into account the fact that the target is moving; iv) we include additional practical constraints such as limited actuation capabilities and the requirement of a finite time to actually lock the gripper and perform the grasp. Although the main focus is on the planning stage, we also discuss how to implement our proposed strategy by closed loop tracking control for the quadrotor motion.

\section{Analysis of new tasks arising in the management of UAVs with a manipulator}

On the basis of the above analysis of existing modern solutions of physical interaction of UAVs with surrounding ground objects, we further formulate the spectrum of new tasks that arise when the UAV is jointly controlled and the manipulator with gripper is installed on it during contact operations with the transported object. In Table 1, new tasks are listed in accordance with the main stages of development and operation of UAVs when manipulating a ground object. At the design stage, it is necessary to calculate the increased values of the UAV parameters and the expansion of the sensor system for manipulating and transporting the object. 
Table 1. New tasks in the implementation of manipulation of UAVs with ground objects.

\begin{tabular}{|c|c|c|}
\hline No. & Task name & demand \\
\hline \multicolumn{3}{|c|}{ At the design stage } \\
\hline 1. & $\begin{array}{l}\text { Selecting the } \\
\text { installation location } \\
\text { of the manipulator } \\
\text { with the gripper on } \\
\text { UAVs }\end{array}$ & $\begin{array}{l}\text { 1. Provide the necessary angles } \\
\text { of motion of the manipulator and } \\
\text { grip for working with objects; } \\
\text { 2. The absence of intersection of } \\
\text { the workspaces of the } \\
\text { manipulator with moving } \\
\text { elements of the UAV. }\end{array}$ \\
\hline 2. & $\begin{array}{l}\text { Selection and } \\
\text { location of the } \\
\text { sensor system }\end{array}$ & $\begin{array}{l}\text { 1. Arrangement of additional } \\
\text { sensors and video cameras to } \\
\text { control the manipulator and grip } \\
\text { when manipulating the object. }\end{array}$ \\
\hline 3. & $\begin{array}{l}\text { The calculation of } \\
\text { the increased } \\
\text { values of } \\
\text { parameters UAV } \\
\text { for transporting } \\
\text { objects }\end{array}$ & $\begin{array}{l}\text { 1. Providing increased flight } \\
\text { performance for a flight with a } \\
\text { manipulator and payload; } \\
\text { 2. Providing additional energy } \\
\text { resources for flight with a } \\
\text { manipulator and payload. }\end{array}$ \\
\hline \multicolumn{3}{|c|}{ On the take-off phase } \\
\hline 4. & $\begin{array}{l}\text { Selecting the } \\
\text { position of the } \\
\text { manipulator }\end{array}$ & $\begin{array}{l}\text { 1. Providing minimal } \\
\text { aerodynamic drag of the } \\
\text { manipulator; } \\
\text { 2. The provision of compact } \\
\text { parking position of the manipulator } \\
\text { with minimal increase in the } \\
\text { dimensions of the UAV; } \\
\text { 3. Ensure the stable position of } \\
\text { UAVS when moving the } \\
\text { manipulator to the parking } \\
\text { position; } \\
\text { 4. Providing fixation of the grip } \\
\text { on the body of the UAV. }\end{array}$ \\
\hline 5. & $\begin{array}{l}\text { The } \\
\text { synchronization of } \\
\text { the work of UAV } \\
\text { and gripping, used } \\
\text { for landing on } \\
\text { ground stationary } \\
\text { objects }\end{array}$ & $\begin{array}{l}\text { 1. Providing a synchronized take- } \\
\text { off and compression of the grip } \\
\text { with the ground object, based on } \\
\text { the analysis of data from the } \\
\text { sensors; } \\
\text { 2. Providing the transfer of the } \\
\text { manipulator to the parking } \\
\text { position. }\end{array}$ \\
\hline \multicolumn{3}{|c|}{ At the stage of object grasp } \\
\hline 6. & $\begin{array}{l}\text { Approach to the } \\
\text { manipulated object }\end{array}$ & $\begin{array}{l}\text { 1. Navigate to the object close } \\
\text { enough for translation of the } \\
\text { manipulator in working } \\
\text { condition; } \\
\text { 2. Maintaining a stable position } \\
\text { of UAVS when moving the } \\
\text { manipulator from the parking } \\
\text { position to the working position. }\end{array}$ \\
\hline 7. & $\begin{array}{l}\text { Pointing the } \\
\text { manipulator on the } \\
\text { object }\end{array}$ & $\begin{array}{l}\text { 1. Providing sensory data capture } \\
\text { position relative to the object and } \\
\text { the surrounding objects based on } \\
\text { the variability of the position UAV; } \\
\text { 2. Providing direct line of sight } \\
\text { of the manipulated object with } \\
\text { the joint movements of the UAV } \\
\text { and the manipulator; } \\
\text { 3. The provision of a sufficient } \\
\text { distance from the UAV to the } \\
\text { surrounding objects; } \\
\text { 4. Maintain a stable position of } \\
\text { the UAV when hovering the } \\
\text { manipulator. }\end{array}$ \\
\hline
\end{tabular}

\begin{tabular}{|c|c|c|}
\hline 8. & Capture an object & $\begin{array}{l}\text { 1. Synchronization of object } \\
\text { capture and stabilization of } \\
\text { UAVs; } \\
\text { 2. Providing takeoff UAV with } \\
\text { an object in the capture; } \\
\text { 3. Transfer of the manipulator to } \\
\text { the optimal position for the } \\
\text { flight, taking into account the } \\
\text { parameters of the transported } \\
\text { object. }\end{array}$ \\
\hline \multicolumn{3}{|c|}{ At the stage of object transportation } \\
\hline 9. & Transportation & $\begin{array}{l}\text { 1. Ensuring the safe navigation of } \\
\text { UAVs taking into account the } \\
\text { dimensions of the transported } \\
\text { object. }\end{array}$ \\
\hline \multicolumn{3}{|c|}{ At the stage of unloading the object } \\
\hline 10. & $\begin{array}{l}\text { The approach to the } \\
\text { unloading site }\end{array}$ & $\begin{array}{l}\text { 1. Navigation UAV to the } \\
\text { unloading site taking into } \\
\text { account the dimensions of the } \\
\text { transported object; } \\
\text { 2. Transfer of the manipulator to } \\
\text { the optimal position for } \\
\text { unloading the transported object. }\end{array}$ \\
\hline 11. & $\begin{array}{l}\text { Placement of the } \\
\text { object on the } \\
\text { landing site }\end{array}$ & $\begin{array}{l}\text { 1. Ensure the stable position of } \\
\text { UAVS hovering manipulator; } \\
\text { 2. Installation of the object in the } \\
\text { given area of the unloading site; } \\
\text { 3. Release of the grip and } \\
\text { retraction of the manipulator } \\
\text { UAV from the object; } \\
\text { 4. Transfer of the manipulator to } \\
\text { the parking position. }\end{array}$ \\
\hline
\end{tabular}

At the take-off phase, it is required to select a compact fixed parking position of the manipulator, which provides minimum aerodynamic resistance, and also to maintain a stable position of the UAV when moving the manipulator to the parking position. In the event that the manipulator was used to land a UAV on a ground facility, it is required to ensure synchronization of the operation of the UAV and the capture during undocking and take-off.

At the stage of grasp an object, the UAV is navigated to the object at a sufficiently close distance to move the manipulator to the working state, pointing the manipulator on the object, capturing and taking off the UAVs with the object. The main difficulty at this stage is the provision of a stable position of the UAV during the movement of the manipulator and accurate capture guidance in the dynamically varying position of the UAV.

At the stage of object transportation, it is necessary to ensure safe navigation of UAVs taking into account the dimensions of the transported object.

At the stage of unloading the object, an approach is made to the unloading area, the manipulator is moved to the optimal position for unloading the transported object and placing the object on the landing site. After the installation of the object in the given area of the unloading area, the grip is extended and the manipulator / UAV is withdrawn from the object. Ensuring a stable position of the UAV allows for more accurate manipulation of the manipulator and installation of the object. The above tasks and requirements allow defining the UAV hardware and software parameters necessary for manipulating and transporting ground objects, as well 
as interaction with ground-based service robotic platforms and other collaborative robots $[1,12,13]$.

\section{Conclusion}

Historically, the investigation of control systems of UAVs was focused on preventing falling and contact with surrounding objects. This was mainly due to the inadequate use of the payload available to the UAV. Until now, UAVs have been used mainly for surveillance and monitoring tasks, for example, in search and rescue missions. Nevertheless, the ability of air vehicles to manipulate and move objects they encounter can significantly expand the types of missions performed by unmanned systems. Flying robots equipped with manipulators can lead to significant changes in transport logistics in near-Earth environments.

To date, the use of UAVs for direct interaction with the environment is still limited due to its instability. A number of studies on this topic have been carried out, but most of them have been performed at the modeling level. Undoubtedly, working with objects using a manipulator mounted on unmanned aerial vehicles and studying the control features of multi-rotor platforms equipped with manipulator grip are promising directions for further research.

The present research was partially supported by state research № AAAA-A16-116033110095-0 and project № 1629-04101-ofi_m of the Russian Foundation for Basic Research.

\section{References}

1. Q.T. Ngo, O.Ya. Solenaja, A.L. Ronzhin, Trudy MAI, 95, (2017)

2. A.A. Ivanov, O.A. Shmakov, SPIIRAS Proceedings. 49, 190-207 (2016)

3. V.G. Gradetsky, I.L. Ermolov, M.M. Knyazkov, E.A. Semyonov, A.N. Sukhanov, Mechanism and
Machine Science, Springer, Heidelberg, 275-281 (2014).

4. C.M. Korpela, T.W. Danko, P.Y. Oh, J Intell Robot Syst., 65, 93-101. (2012)

5. T.W. Danko, P.Y. Oh, J Intell Robot Syst., 73, 709723 (2014)

6. S. Gardecki, A. Kasiński, A. Bondyra, P. Gạsior, International Conference Automation, 357-366 (2017)

7. A. Suarez, G. Heredia, A. Ollero, Robot 2015: Second Iberian Robotics Conference, 543-555 (2015)

8. M. Orsag, C. Korpela, P. Oh, J Intell Robot Syst,. 69, 227-240 (2013)

9. A. Khalifa, M. Fanni, International Journal of Control, Automation and Systems 15 (3), 1434 1446 (2017).

10. M. Kobilarov, J Intell Robot Syst., 73, 679-692 (2014)

11. G. Chmaj, T. Buratowski, T. Uhl, K. Seweryn, M. Banaszkiewicz, Aerospace Robotics, 109-119 (2013)

12. A.S. Kodyakov, N.A. Pavlyuk, V.Yu. Budkov, R.A. Prakapovich, Journal of Physics: Conference Series, 803 (1), 012074 (2017)

13. N.E. Shlyakhov, I.V. Vatamaniuk, A.L. Ronzhin, Journal of Physics: Conference Series, 803 (1), 012146 (2017)

14. S Kim., S. Choi, H.J. Kim, 2013 IEEE/RSJ International Conference on Intelligent Robots and Systems (IROS), 4990-4995 (2013)

15. R. Spica, A Franchi., G. Oriolo, H.H. Bülthoff, P.R. Giordano, 2012 IEEE/RSJ International Conference on Intelligent Robots and Systems (IROS), 4985-4992 (2012) 